Gastroenterologe

https://doi.org/10.1007/s11377-019-0318-5

(c) Springer Medizin Verlag GmbH, ein Teil von Springer Nature 2019

CrossMark

Stefan Zeuzem ${ }^{1}$ Jürgen F. Riemann ${ }^{2}$

'Zentrum für Innere Medizin, Medizinische Klinik 1, Universitätsklinikum Frankfurt a.M., Frankfurt a.M., Deutschland

${ }_{2}^{2}$ Medizinische Klinik C, Klinikum Ludwigshafen, Stiftung LebensBlicke, Ludwigshafen, Deutschland

\title{
Erratum zu: Neue Wege in der Darmkrebsprävention
}

Erratum zu:

Gastroenterologe 2018

https://doi.org/10.1007/s11377-018-

0308-z

Nach der Online-first-Publikation der „Einführung zum Thema" hat sich eine wichtige Änderung ergeben, die eine Aktualisierung des Inhalts zur Folge hatte. Bitte beachten Sie die korrekte, im Heft veröffentlichte und nun auch online verfügbare Version.

Der Originalbeitrag wurde korrigiert.

Die Redaktion

\section{Korrespondenzadresse}

Prof. Dr. Stefan Zeuzem

Zentrum für Innere Medizin, Medizinische Klinik 1, Universitätsklinikum Frankfurt a.M. Theodor-Stern-Kai 7, 60590 Frankfurt a.M., Deutschland

zeuzem@em.uni-frankfurt.de

\section{Prof. Dr. Jürgen F. Riemann}

Medizinische Klinik C, Klinikum Ludwigshafen, Stiftung LebensBlicke

Parkstr. 49, 67061 Ludwigshafen, Deutschland riemannj@garps.de

Prof. Dr. Jürgen F. Riemann ehem. Medizinische Klinik C, Klinikum Ludwigshafen, Stiftung LebensBlicke,

Parkstr. 49, 67061 Ludwigshafen, Deutschland 